ISSUES IN MEDICINE

\title{
Haemorrhage associated with caesarean section in South Africa - be aware
}

\author{
S Fawcus, J Moodley, for the National Committee on Confidential Enquiries into Maternal Deaths (NCCEMD)
}

The National Committee for Confidential Enquiries into Maternal Deaths (NCCEMD), which since 1998 has produced triennial reports of maternal deaths in South Africa, has identified the increasing rate of haemorrhage during and after caesarean section (CS) as a problem. Obstetric haemorrhage is the third most common cause of maternal death, accounting for 491 of the total 3959 deaths in 2005 - 2007, but constitutes one of the most avoidable causes of maternal death, over $80 \%$ of cases being thought to be 'clearly avoidable'.

Deaths from obstetric haemorrhage are further categorised as follows: abruptio placentae, placenta praevia, uterine atony, retained placenta, uterine rupture, uterine inversion and other uterine trauma'. This latter group predominantly comprises severe bleeding during and/or after CS, and is the largest group, accounting for 141 deaths $(28.7 \%$ of all haemorrhage-related maternal mortality) in 2005 - 2007. ${ }^{1}$ Of concern is that this has increased compared with 2002 - 2004, when it accounted for 78 deaths (17.6\% of haemorrhage mortality). Also, some deaths classified under other causal groups such as uterine atony, abruptio placentae and placenta praevia were due to severe bleeding at CS. This gives a total of 201 maternal deaths during 2005 - 2007 with severe bleeding at CS (41\% of the total deaths from obstetric haemorrhage). During 2005 - 2007 a total of 477210 CSs were performed in public sector facilities; $35 \%$ at district hospitals, $40 \%$ at regional hospitals and $25 \%$ at tertiary hospitals, giving a national public sector CS rate of $18.4 \%$ (District Health Information Systems data).

Therefore, of a total of 477210 women who underwent CS during the years 2005 - 2007, 201 died from haemorrhage at CS, giving a CS haemorrhage fatality rate of $0.042 \%$ (4.2 deaths from haemorrhage at CS for every 10000 CSs performed). Further analysis of 103 of these deaths revealed the following:

- Of the CSs $92.2 \%$ were emergencies, compared with $7.8 \%$ elective operations.

- Of the CSs $37.9 \%$ were performed at district, $41.7 \%$ at regional and $20.4 \%$ at tertiary hospitals.

- The commonest indications for emergency CS were obstructed labour, abruptio placentae, patient with a previous CS presenting in labour, placenta praevia and fetal distress.

$J$ Moodley is Professor Emeritus in the Department of Obstetrics and Gynaecology, University of KwaZulu-Natal. Professor Fawcus is Head of the Department of Obstetrics and Gynaecology, Mowbray Hospital, Cape Town, and an Associate Professor in the Department of Obstetrics and Gynaecology, University of Cape Town.

Members of the NCCEMD: D M Chweneyagae, S Fawcus, $R$ Fuentes, $N$ Godi, $N$ Khaole, B Kunene, $M$ Lekhoathi, M Masasa, $Z$ Mbambisa, $N$ Mbombo, $R$ E Mhlanga, $N$ Molefe, $R$ Molupe, J Moodley, N Moran, R C Pattinson, C Rout, R Seabe, M Schoon, J Sithole.
- In many cases, postoperative bleeding followed intra-operative problems with haemostasis, frequently due to an atonic uterus or uterine tears from a distended lower segment after difficult delivery of an impacted head. In a few cases the bleeding originated from the placental bed, such as in placenta praevia or previous CS, and in some surgery was difficult due to multiple adhesions.

- Twenty-seven per cent had a hysterectomy, either at initial CS or at re-laparotomy; $5 \%$ had a re-laparotomy but no hysterectomy. Of great concern is that $68 \%$ of the total group had no additional conservative surgical measures, no re-look laparotomy and no hysterectomy.

- Blood was transfused in $70 \%$ of cases, no blood was given in $28 \%$, and $2 \%$ declined blood products.

- Deaths were assessed as clearly avoidable in $85 \%$ of cases. Lack of and insufficient blood was a problem in $28 \%$, and staff shortages, particularly for postoperative monitoring, were a factor in $15.5 \%$. Non-functioning theatres at level 1 were a problem for some, and delayed access to theatre due to other emergencies occurred in $6 \%$ of cases at level 2 and 3 hospitals. Deficiencies in clinical care, including poor monitoring in the postoperative period (35\%), were identified in most cases and led to serious delays in detecting haemorrhagic shock, poor resuscitation (17.5\%) and incorrect management $(7.8 \%)$. There was grossly substandard care in $60 \%$ of cases, including failure to secure haemostasis at the initial CS, non-use of potentially effective treatment such as parenteral ergometrine, uterine compression sutures and balloon tamponade, and delays in definitive management such as hysterectomy.

Over the past few months several deaths associated with bleeding during and immediately after CS have been reported, despite dissemination of information on the prevention of deaths from obstetric haemorrhage.

\section{Towards solving the problem}

Knowledge and clinical skills in resuscitation, clinical decisionmaking, clinical interpretation and surgical techniques must be improved. Teamwork and administrative support are also essential to prevent and manage severe bleeding associated with CS.

The Health Professions Council of South Africa (HPCSA) stipulation of 4 months in obstetrics and gynaecology during the extended 2-year internship, and the logbook requirement for training in CS, are positive developments. Strengthening ongoing training and ensuring that the logbook requirements are fulfilled require more attention.

The Essential Steps in Management of Obstetric Emergencies $(\mathrm{ESMOE})^{2}$ training programme for interns includes surgical skills with an emphasis on CS. The 'Haemorrhage' module has algorithms for medical treatment of uterine atony and additional surgical measures to arrest haemorrhage at CS.

The NCCEMD Monograph on the Management of Postpartum Haemorrhage is a practical pocket book for health workers at all levels of care. ${ }^{3}$ It has a section on the prevention and management of severe blood loss at and after CS, with algorithms. There are also posters on the management of postpartum haemorrhage (PPH) for labour wards, and on B lynch compression sutures and uterine artery ligation for theatres. 


\section{Algorithms for bleeding associated with caesarean section}

Fig. 1 is an algorithm for bleeding detected during CS, and Fig. 2 an algorithm for bleeding diagnosed in the postoperative period.

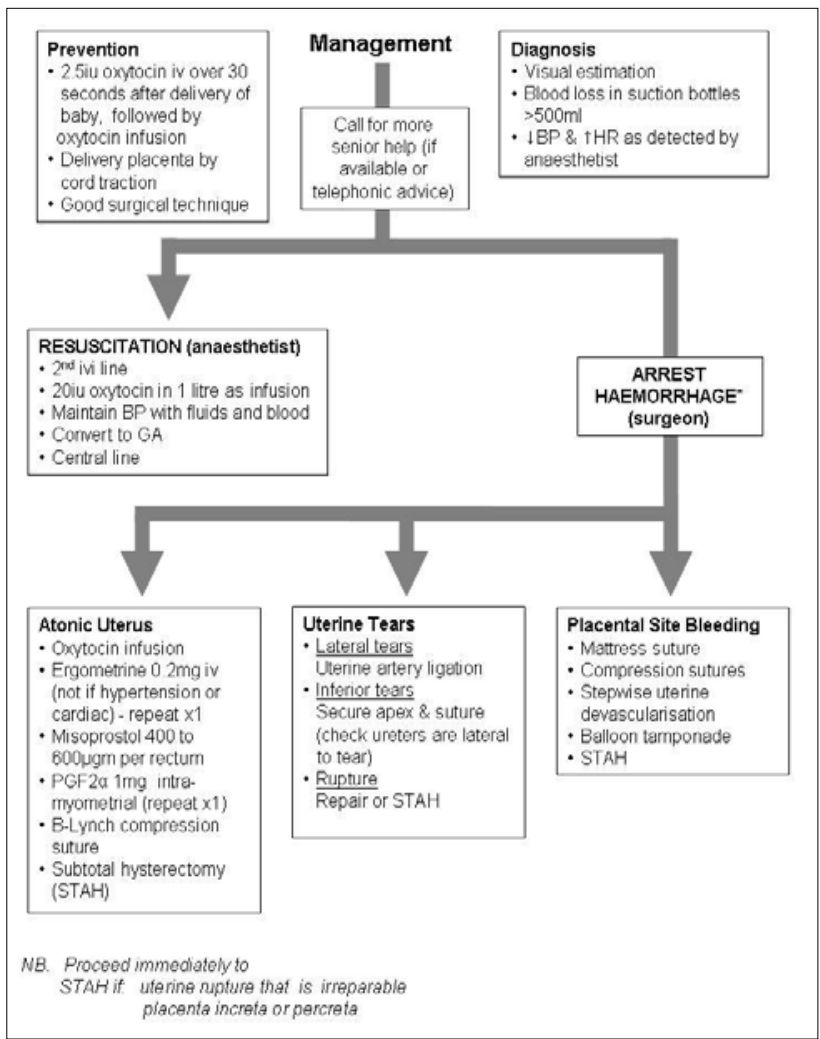

Fig. 1. Management of bleeding at caesarean section (STAH = subtotal abdominal haemorrhage).

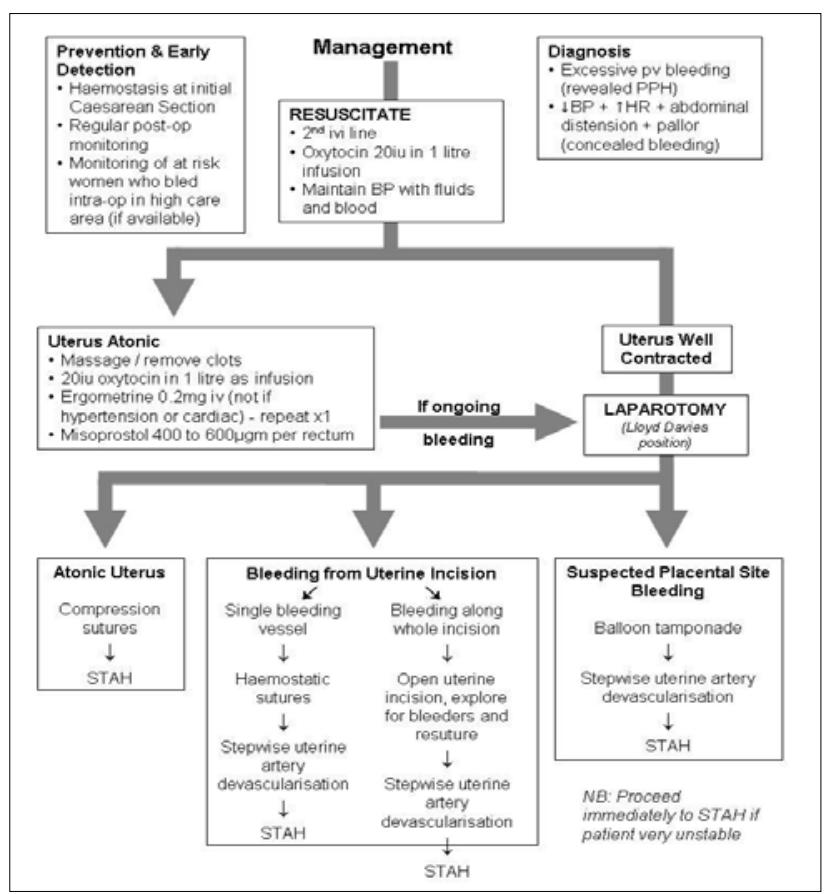

Fig. 2. Management of bleeding after caesarean section (STAH = subtotal abdominal haemorrhage).

\section{Abdominal hysterectomy}

Proceed straight to hysterectomy in cases of placenta percreta, a ruptured uterus that is irreparable, or when conservative measures are unsuccessful. A subtotal abdominal hysterectomy (STAH) is usually sufficient to control the bleeding unless there are tears down into the cervix or in some cases of lower segment bleeding following a major placenta praevia, when the cervix must also be removed, i.e. a total abdominal hysterectomy (TAH) must be performed.

If haemostasis is not satisfactory after STAH, a suction drain can be left in situ. If coagulopathy is evident after the STAH/TAH, consider abdominal packing to tamponade the abdominal cavity. At least 5 paediatric swabs followed by abdominal swabs can be used. The patient must be kept ventilated and the packs removed after $24-48$ hours.

\section{Level of care}

Stepwise medical treatment of uterine atony, uterine compression sutures, balloon tamponade and uterine artery ligations are skills that must be learnt by doctors performing CS and can be done in a level 1 hospital with emergency blood available. STAH skill may not be available at level 1 hospitals, but should be available at all level 2 and 3 hospitals, which are more appropriate to manage patients with massive haemorrhage

\section{What can we do to reduce the frequency of this problem?}

Algorithms, posters and booklets distributed to the relevant health workers are useful, but are insufficient on their own. 'Hands-on' surgical training for all doctors performing CS, and an approach to and demonstration of additional surgical skills to arrest excessive bleeding, must be emphasised. Experienced specialist assistance for difficult cases, preferably on site or in the form of easily available telephonic advice for remote rural hospitals, is also important. Clinical outreach can help maintain skills and surgical training.

Clinical managers of maternity services should monitor bleeding associated with CS as an indicator, and ensure that guidelines are in place, that surgical training occurs and that experienced assistance is available.

Prevention is important, e.g. earlier recourse to CS in cases of prolonged labour and the use of oxytocics before removal of the placenta. Early detection of postoperative bleeding enables earlier treatment. It is important to recognise that postoperative CS haemorrhage may be concealed because the bleeding is intraabdominal.

The NCCEMD sent the following recommendation to all heads of institutions and training schools involved in maternity care:

The following steps should be taken to minimise haemorrhage associated with CS:

- Ensure not only that the partogram is completed fully but that the labour recordings are interpreted properly, in order for prolonged labour to be diagnosed timeously, the appropriate interventions made, and late first- and secondstage CS avoided.

- Ensure that the strict indications for CS are followed. If you know in advance that a patient has a major placenta praevia, she should be referred to a second level or tertiary hospital for further management.

- Ensure that the haemoglobin level is known before CS, and if it is $<8 \mathrm{~g} / \mathrm{dl}$ ensure that blood is available. 
- If the CS is for the indication of prolonged labour/obstructed labour, ensure that the most experienced medical officer performs the surgery.

- Ensure that there is a dedicated medical officer/specialist to provide anaesthesia, i.e. at least two doctors should be available for the procedure, one to provide the anaesthesia and one to do the surgery. In emergency situations, an experienced midwife can assist at the surgery.

- Ensure that appropriate surgical technique is used and that the incision in the uterus is not too low in the lower uterine segment, particularly in CS for prolonged labour and in the second stage of labour.

- DO NOT RUSH THE SURGICAL PROCEDURE. A CS should take about 30 - 40 minutes.

- Do not close the abdomen before checking that haemostasis has been achieved and that the uterus is contracted. Also check with the anaesthetist that the patient is cardiovascularly stable; the blood pressure should be $>100 \mathrm{mmHg}$. Intra-operative resuscitation with the abdomen open should be done until this figure is reached. If intra-operative bleeding persists, call for help.

- Ensure that after delivery of the baby, prophylactic oxytocin (Syntocinon) $2.5 \mathrm{IU}$ is given by slow IV injection; 10 units is added to one litre of fluid and continues to 'run' during the operation, after the operation in the recovery room, and in the ward for at least 2 hours. Do not stop the oxytocin drip at any stage during transfer of the patient from the theatre to the ward. For treatment of uterine atonicity, 20 IU of oxytocin is added to one litre of fluid.

- Ensure that before the patient leaves the operating table and the recovery room, the uterus is palpated to confirm that it is not atonic. Also check to assess the amount of vaginal bleeding and empty the uterus of all blood clots.

- Transfer the patient from the operating theatre/recovery room only after ensuring that the pulse rate and blood pressure are stable and that the spinal is $<\mathrm{T} 8$ and receding. Also check for uterine/vaginal bleeding ('pad checks').

- Ensure clinical observations in the following manner:

- half-hourly for 2 hours

- hourly for a further 4 hours

- 2-hourly for a further 6 hours and then 4-hourly until discharge from hospital.

- Observations to be recorded:

- pulse rate

- blood pressure

- respiratory rate

- vaginal bleeding (pad checks)

- urine output 4-hourly.

- The patient should be nursed in an area where there are health care professionals who can do the observations regularly, and not be placed in a ward where observations are difficult to perform.

Your help in following the abovementioned steps will ensure that bleeding associated with CS is minimised.
For a more detailed approach to the management of bleeding associated with CS, see the PPH Monograph (pages 21 - 24), available free of charge from the NDOH or your provincial Maternal Child and Women's Health Cluster. The information can also be obtained from the website www.doh.gov.za

1. National Committee on Confidential Enquiries into Maternal Deaths. Saving Mothers. Fourth Report on Confidential Enquiries into Maternal Deaths in South Africa 2005-2007. Pretoria: Department of Health, 2009.

2. National Committee on Confidential Enquiries into Maternal Deaths. Saving Mothers. Essential Steps in the Management of Common Conditions Associated with Maternal Mortality. Pretoria: Department of Health, 2007.

3. National Committee on Confidential Enquiries into Maternal Deaths. Monograph on the Management of Postpartum Haemorrhage. Pretoria: Department of Health, 2010. 